\title{
Chromosome Morphology and Meiosis in Some Eu-sorghums
}

\author{
M. L. Magoon, K. G. Shambulingappa and M. S. Ramanna \\ Division of Botany, Indian Agricultural Research Institute, \\ New Dalhi, India
}

Received March 7, 1961

\section{Introduction}

The comparison of the chromosome morphology and the process of meiosis in different taxa is one of the ways of arriving at an estimate of the relationship between such different taxonomic entities, especially between species and lower categories. This approach has, however, been exploited rather infrequently, perphaps because of the technical difficulties inherent in it. In particular, even though the pioneering investigations of McClintock showed, as early as 1929, the wealth of morphological criteria which can be obtained from an analysis of the pachytene of meiosis, studies on the comparative morphology of chromosomes at this stage have, until recently, been few (see Magoon and Shambulingappa 1960).

The sorghums, which play an important role in many of the tropical countries are an extremely variable group. There has, consequently, been considerable disagreement as to the classification to be adopted in this group and to the taxonomic status to be accorded to different forms as also regarding the evolutionary relationship between them. It, therefore, appears necessary that as much information as possible should be obtained regarding the different taxa included in this highly complex genus. It is the purpose of this paper to compare the karyomorphology of five species which have been classified under the sub-genus Eu-Sorghum.

\section{Material and methods}

The following wild and cultivated types were used in the present study.

1. S. verticilliflorum, Wild. P. 1. 208190

2. S. arundinaceum, Wild. P. 1. 208573

3. S. dochna, Cultivated P. 1. 246592

4. S. saccharatum, Cultivated E. C. 11608

5. S. caudatum, Cultivated I. W. 11111

Seeds of these species were obtained through the Rockefeller Foundation, Botany Division, I.A.R.I., New Delhi and were sown under field conditions. For the study of chromosome morphology and meiosis, the simple 
propiono-carmine smear method (Swaminathan et al. 1954 and Magoon et al. 1958) was followed.

Different methods of depicting the pachytene chromosomes have been followed depending mostly on the type of the pachytene chromosomes. Since in this material the pachytene chromosomes proved to be somewhat similar to that of tomato, as suggested by Barton (1950) and Gottschalk (1954), straight line drawings of the different regions were made from photomicrograph and such drawings used for measuring the length etc. For the sake of convenience in comparing the chromosome sizes "Relative length" was used which represents the ratio in percentage of the length of the individual chromosome to that of the longest in the complement (Huziwara 1956).

\section{Observations}

Chromosome morphology

Pre-pachytene stages are not easily amenable for critical analysis. However, observations were recorded in several nuclei in each of these species at mid pachytene (Figs. 1-5). A characteristic feature noticed uniformly in all the species studied is that the centromere of each of the ten pachytene chromosomes could be easily distinguished as an oval shaped, achromatic structure flanked by segments composed of deeply staining chromomeres followed by a lightly staining region. The proximal, chromomeric regions appear to have a great thickness than the achromatic regions. This difference in thickness is particularly marked in the case of $S$. verticilliflorum.

The following characters alone or in combination can be used in the identification of the individual pachytene bivalent. 1) total length, 2) relative length, 3) chromomeric patterns, 4) position of the centromere, 5) presence of deeply staining knobs, 6) relative length of chromatic and achromatic portions of each arm, and 7) presence or absence of deeply staining chromomeres close to the chromatic region.

In the absence of other criteria, total length, relative length, the centromeric position and the presence or absence of deeply staining chromomeres close to the chromatic region have been considered here in depicting the pachytene chromosomes. Based on the average values obtained by accurate measurements of the chromosomes in several nuclei where all the chromosomes could be traced from end to end, the ten pachytene bivalents of each species have been arranged in the order of their length and numbered in decreasing order of length, chromosome I being the longest and chromosome $\mathrm{X}$ being the shortest. Their total length, relative length and arm ratio are presented in Table 1.

Principal diagnostic morphological features of each of the ten chromosomes in the haploid complement of the five species studied are briefly noted below :- 
Table 1. The average values of length in microns of the pachytene complement in the five species

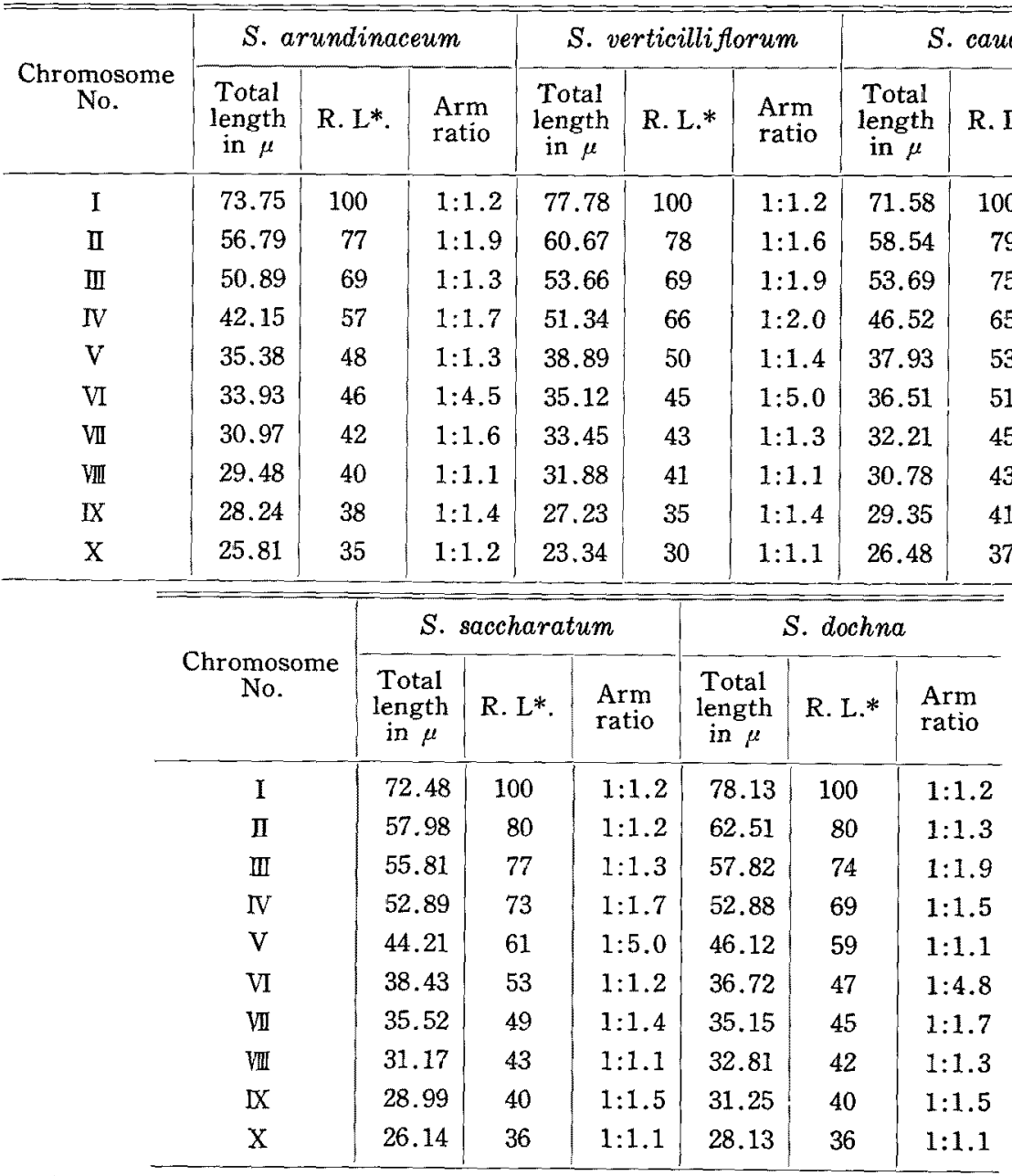

R. L. $=$ Relative length

\section{1) S. arundinaceum}

Chromosome I: Longest of the complement measuring on an average $73.75 \mu$; with a nucleolar organizing region in the long arm (unlike in the other species where this region is on the short arm) lying very close to the centromere. On the long arm, two deeply stained regions follow the nucleolar organizing region. The deeply staining region ends abruptly on the short arm. The short arm has a terminal chromomere. The arm ratio is $1: 1.2$.

Chromosome II: Measures about $56.79 \mu$ with an arm ratio of $1: 1.9$. A series of deeply staining chromomeres are present after the deeply staining region on the short arm. Both the arms have terminal chromomeres. On the long arm, the deeply staining region ends abruptly.

Chromosome III : This is very close to the second chromosome in its total length $(50.89 \mu)$ but shows a marked difference in the arm ratio $(1: 1.3)$. 
The deeply staining region is followed by a deeply staining chromomere on the short arm but ends suddenly on the long arm.

Chromosome IV: This can be differentiated from the preceding chrosomes on the basis of average length $(42.15 \mu)$ as well as arm ratio, the latter being $1: 1.7$. The deeply staining region on both the arms is of almost equal length and ends abruptly.

Chromosome $\mathrm{V}$ : This belongs to the short chromosome group measur. ing about $35.38 \mu$ and having an arm ratio of $1: 1.3$. The deeply staining region is short on the short arm and is immediately followed by a deeply staining chromomere on this arm while it is longer on the long arm. This arm terminates in a chromomere.

Chromosome VI: Measures about $33.93 \mu$. This is readily identified in the complement by its great asymmetry, the ratio between the arms length being $1: 4.5$. The deeply staining region on the long arm ends suddenly. About $3 / 4$ th of the short arm stains deeply, and is followed by $2-3$ chromomeres.

Chromosome VII : Measures about $30.97 \mu$ with an arm ratio of $1: 1.6$. This chromosome can also be distinguished by the much greater length of the deeply staining region on the short arm as compared to that on the long arm.

Chromosome VIII : Measures about $29.48 \mu$ and has an arm ratio of $1: 1.1$. This chromosome can be readily distinguished from the rest of the complement because of the nearly equal arms. The short arm has two deeply staining regions, the former being longer and followed by a smaller one. The deeply staining region on the long arm ends abruptly.

Chromosome IX: Measures about $28.24 \mu$ and has an arm ratio of $1: 1.4$. The deeply staining region on the short arm ends abruptly while on the long arm it is followed by a chromomere.

Chromosome X: Shortest chromosome of the complement measuring about $25.81 \mu$. Both the arms are of almost equal in their length, the arm ratio being $1: 1.2$. A series of chromomeres on both the arms follow the deeply staining region. Their number varies-being larger on the longer arm and smaller on the shorter arm.

\section{2) S. verticilliflorum}

Chromosome I: Longest of the complement measuring on an average $77.78 \mu$ with nucleolus organizer on the short arm which ends in a terminal chromomere. The arm ratio is $1: 1.2$. The darkly staining region on the long arm ends abruptly. On the short arm, a darkly staining region, following the nucleolar organizer which is comparatively larger than an ordinary chromomere, is present.

Chromosome II : Measures about $60.67 \mu$ with an arm ratio of $1: 1.6$. The short arm has a longer deeply staining region than is the longer arm. While a chromomere on the short arm is present a little distance away from 
the deeply staining region, it is located on the long arm very close to the darkly staining region.

Chromosome III : Measures on an average $53.66 \mu$. This and the IV chromosome are almost of equal length and have a similar arm ratio $(1: 2)$. However, the identification of this chromosome is facilitated by the fact that the deeply staining region is smaller on the short arm and longer on the

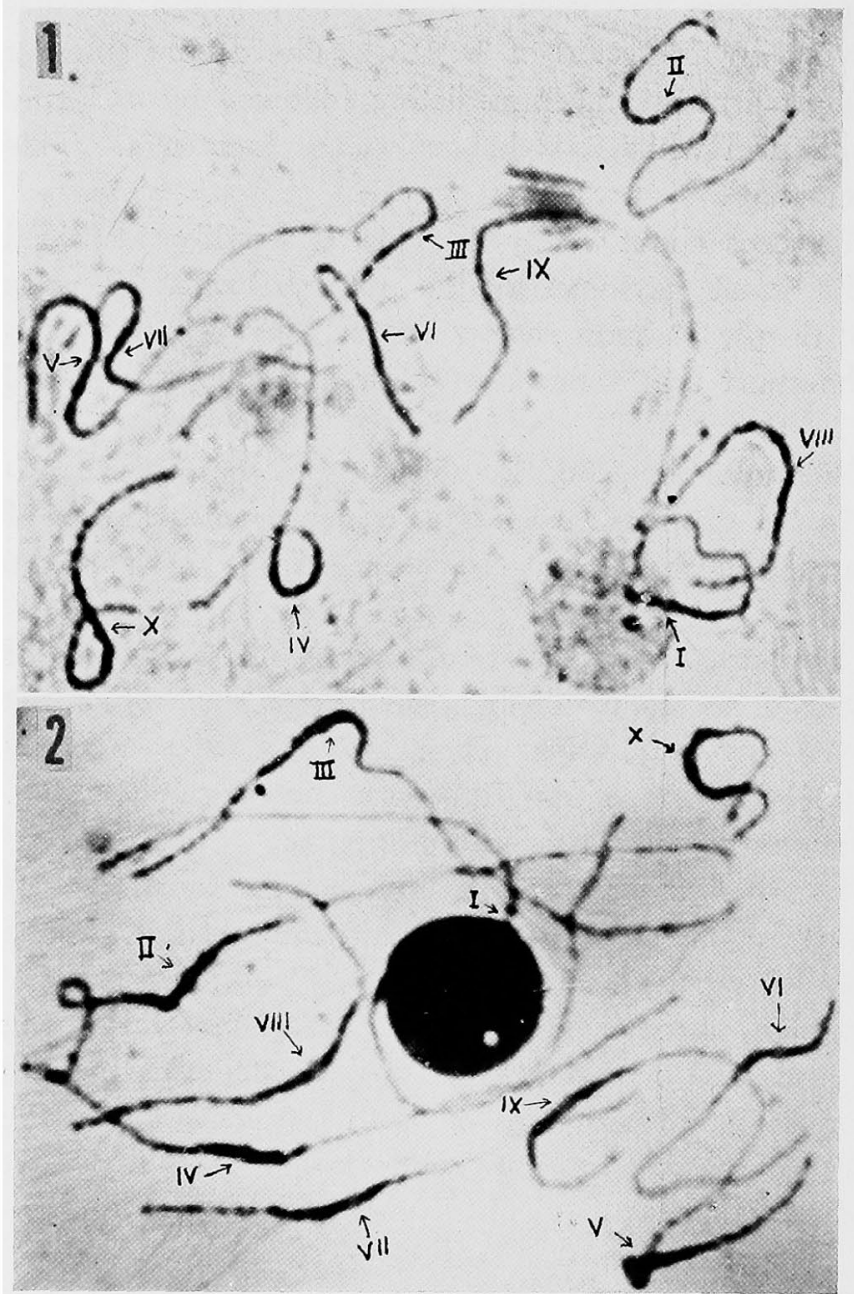

Figs, 1-2. Pachytene chromosomes of S. arundinaceum and $S$. verticilliflorum respectively numbered according to their length. Arrows indicate the position of centromeres (ca. $\times 1000$ and $\times 1200$ respectively). long arm. While the darkly staining region on the long arm ends abruptly, it is followed by another deeply staining region and a chromomere on the short arm.

Chromosome IV : This chromosome is $51.34 \mu$ long with an arm ratio of 1.2 . The deeply staining regions on both the arm are almost equal. But it ends abruptly on the short arm, whereas on the long arm it is followed by a chromomere. The short arm bears a terminal chromomere.

Chromosome V: Measures about 38.89 $\mu$, having an arm ratio of $1: 1.4$. This can be easily identified from the preceding chromosomes on the basis of its comparatively shorter in length while it differs from the shorter chromosomes in the chromomeric pattern. The deeply staining region on the long arm ends abruptly but on the short arm it is followed by 2-3 chromomeres.

Chromosome VI : Measures about $35.12 \mu$. The quick identification of this chromosome in the complement is facilitated by its characteristic a-symmetry, arm ratio being 1:5 i.e., the long arm is about 5 times the length 
of the short arm. The deeply staining region ends abruptly on the long arm while about $3 / 4$ th of the short arm stains deeply and 1 or 2 chromomeres are noticed at the terminal region.

Chromosome VII : Measures about $33.45 \mu$ with an arm ratio of $1: 1.3$. The darkly staining region is smaller on the long arm as compared to that on the short arm. It ends abruptly on the long arm, while on the short arm, it is followed by two big and 1 or 2 small chromomeres.

Chromosome VIII: Measures on an average $31.88 \mu$. This can be easily picked out from the rest of the complement because of its great symmetry, with an arm ratio of $1: 1.1$. On one arm, there are two darkly staining regions which are very close to each other, the proximal one having a greater thickness than the distal one. The deeply staining region on relatively shorter arm ends abruptly. It has a terminal chromomere.

Chromosome IX: Measures on an average $27.23 \mu$. Arm ratio is $1: 1.4$. The darkly staining region on both the arms are almost equal and they are followed by a comparatively lightly staining region.

Chromosome $\mathrm{X}$ : This is the shortest chromosome of the complement measuring about $23.34 \mu$. This is another symmetrical form in the comple. ment having an arm ratio of $1: 1.1$. However, this chromosome can be distinguished from the 8 th chromosome (which is also symmetrical) on the basis of total average length and the distribution of darkly staining regions. Nearly about $50 \%$ of the length of this chromosome is pycnotic in character. Thus, it has a very large staining region in relation to its length when compared to the other chromosomes.

\section{3) S. caudatum}

Chromosome I : Longest chromosome in the complement, measuring on an average $71.58 \mu$ and having an arm ratio of $1: 1.2$. The nucleolar organizing region is located on the short arm and no other deeply staining regions or chromomeres are present following this region. The pycnotic region ends abruptly on the long arm.

Chromosome II : Measures on an average $58.54 \mu$, with an arm ratio of $1: 1.4$. The deeply staining region on the short arm is longer than on the long arm. Whereas on the long arm it is followed by a deeply staining chromomere, on the short arm it ends abruptly.

Chromosome III : Measures about $53.69 \mu$. It is difficult to separate out this chromosome from chromosome II because of their almost similar total length. However, arm-ratio, which is almost $1: 2$, in addition to chromomeric pattern can be conveniently used to differentiate between these two chromosomes. The deeply staining regions on both the arms are almost equal and whereas the pycnotic region ends abruptly on the long arm, it is followed by a chromomere on the short arm.

Chromosome IV: Measures on an average $46.52 \mu$ with an arm-ratio of $1: 1.5$. The deeply staining region which is almost equal on both the 
arms, is followed by a deeply staining chromomere on the long arm, while it ends abruptly on the short arm.

Chromosome V: This chromosome is very similar to the chromosome IV in arm ratio, being $1: 1.5$ in each case. However, it differs in its shortness (average length $37.93 \mu$ ) in addition to chromomeric pattern. There are two deeply staining regions on the short arm, very close to each other,

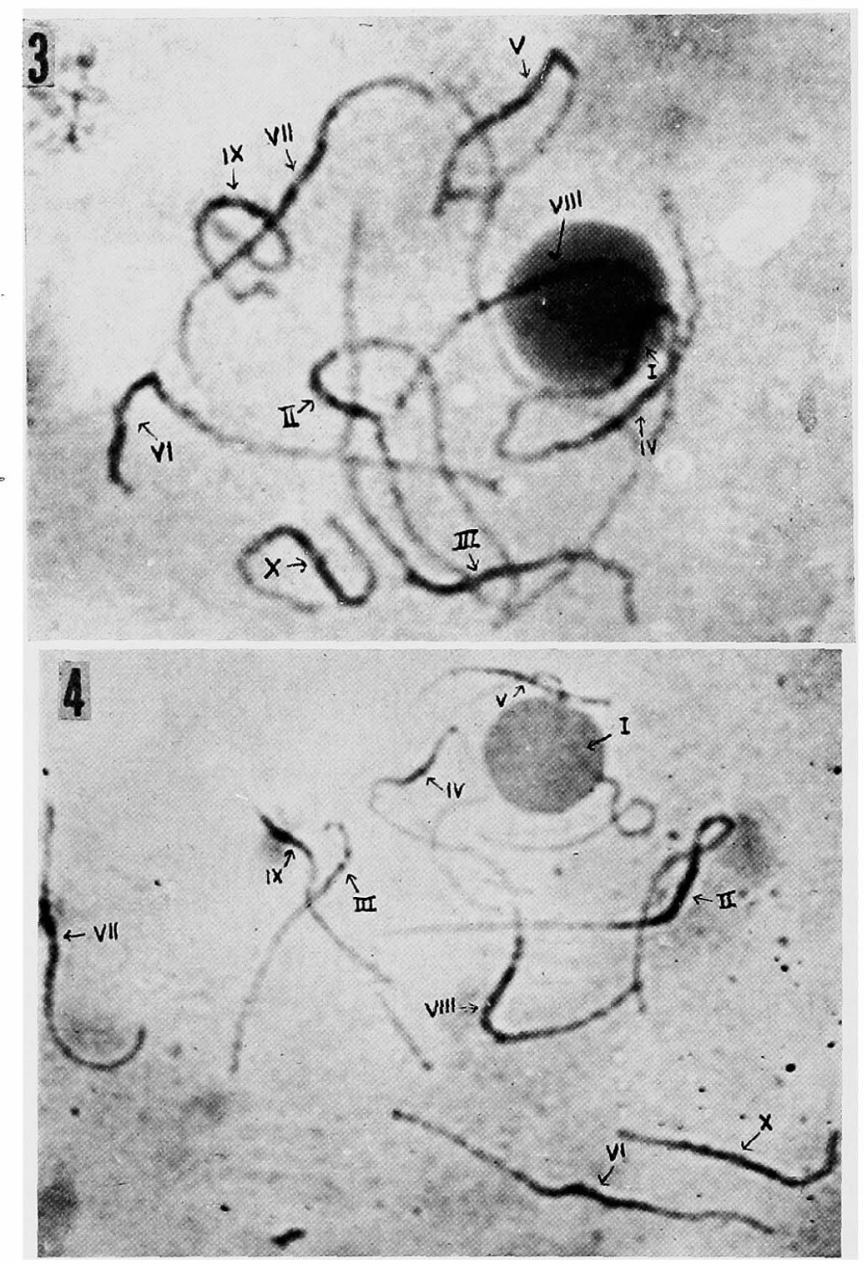

Figs. 3-4. Pachytene chromosomes of S. caudatum and $S$. saccharatum respectively $(\times 1200$ and $c a . \times 1000$ respectively). while on the long arm it ends abruptly.

Chromosome VI:

Measures about $36.51 \mu$. It can be easily picked out in the complement because of the great asymmetry; the arm ratio being $1: 5$. The short arm stains dark almost completely leaving a short terminal region unstained except for a deeply stained chromomere. The pycnotic region on the long arm ends abruptly.

Chromosome VII : Measures on an average $32.21 \mu$ with the arm ratio of $1: 1.2$. Since the remaining three chromosomes are quite similar to this chromosome in their length, the separation and identification of each one has to be based on chromomeric pattern. The chromosome under consideration has a longer pycnotic region on the long arm and a shorter such region on the short arm is followed on both the arms by a deeply staining chromomere.

Chromosome VIII : Measures about $30.78 \mu$. This chromosome has a symmetrical form having an arm ratio almost $1: 1.1$. One arm has a large deeply staining region which ends abruptly and with a terminal chromomere 
and the deeply staining region on the other arm is comparatively shorter and is followed by two chromomeres.

Chromosome IX: Measures about $29.35 \mu$, with an arm ratio of $1: 1.3$. The short arm has two darkly staining regions towards the proximal end which are very close to one another. The long arm has a deeply staining chromomere following the pycnotic region.

Chromosome $\mathrm{X}$ : This is the shortest chromosome of the complement measuring about $26.48 \mu$ and has an arm ratio of $1: 1.1$. There are $2-3$ deeply staining chromomeres following the pycnotic region on both the arms.

\section{4) S. saccharatum}

Chromosome I: It is the longest of the complement measuring about $72.48 \mu$ having an arm ratio of $1: 1.2$. Nucleolus organizer is situated on the short arm. The long arm has a deeply staining chromomere following the deeply staining region.

Chromosome II : Measures on an average $57.98 \mu$, the arm ratio being 1:1.2. The darkly staining regions on both the arms are equal and are followed by two chromomeres on the short arm and one on the long arm.

Chromosome III : Measures about $55.81 \mu$ with an arm ratio of $1: 1.3$. It is often very difficult to separate out this chromosome II because of the fact that both the chromosomes are very similar in length and arm ratio. However, the darkly staining region on the short arm ends absuptly in this chromosome, while on the long arm, it is followed by a deeply staining chromomere.

Chromosome IV: Measures about $52.89 \mu$-almost the same length as that of chromosomes II and III but this can be easily differentiated from them on the basis of arm ratio $(1: 1.7)$ and chromomeric pattern. The long arm has a chromomere following the pycnotic region whereas on the short arm this ends abruptly.

Chromosome $\mathrm{V}$ : This most asymmetrical form of chromosome measuring about $44.21 \mu$ with an arm ratio of $1: 5$ occupies the fifth place in this species whereas in the other species the asymmetrical chromosome is the sixth one. In morphology, it resembles the most asymmetrical form of chromosomes found in the other species. The short arm stains deeply for about $3 / 4$ th of its length having 1 or 2 chromomeres at the terminal end. The darkly staining region ends abruptly on the long arm.

Chromosome VI : Measures about $38.43 \mu$, with an arm ratio of $1: 1.2$. The darkly staining region is more on the short arm than on the long arm and is followed by one chromomere on the short arm and by two chromo. meres on the long arm.

Chromosome VII : Measures about $35.52 \mu$ having an arm ratio of $1: 1.4$. The pycnotic region on the long arm is longer than on the short arm and ends abruptly whereas it is followed by a chromomere close to the pycnotic region on the short arm. 
Chromosome VIII : Measures about $31.17 \mu$ having an arm ratio of $1: 1.1-$ a very symmetrical chromosome as in the other species. The long arm has in addition to darkly staining region two chromomeres, one terminal and the other very close to the pycnotic region. On the other hand following the pycnotic region there are two chromomeres which are very close together.

Chromosome IX: Measures about $28.99 \mu$ with an arm ratio of $1: 1.5$. The deeply staining region on the short arm which ends abruptly is larger than on the long arm where it is followed by a chromomere.

Chromosome $\mathrm{X}$ : This is the shortest and another very symmetrical form in the complement measuring about $26.14 \mu$ and having an arm ratio of $1: 1.1$. As in other species, the pycnotic regions on both the arms are followed by $2-3$ chromomeres.

5) S. dochna

Chromosome I: It is the longest in the complement measuring about $78.13 \mu$. The arm ratio being $1: 1.2$. The nucleolar organizer is situated on the short arm and is followed by two chromomeres. The pycnotic region on the long arm ends abruply.

Chromosome II : Measures on an average $62.51 \mu$, having an arm ratio of $1: 1.3$. Following the darkly staining region there are two chromomeres on the short arm. Only one chromomere is present on the long arm follow.

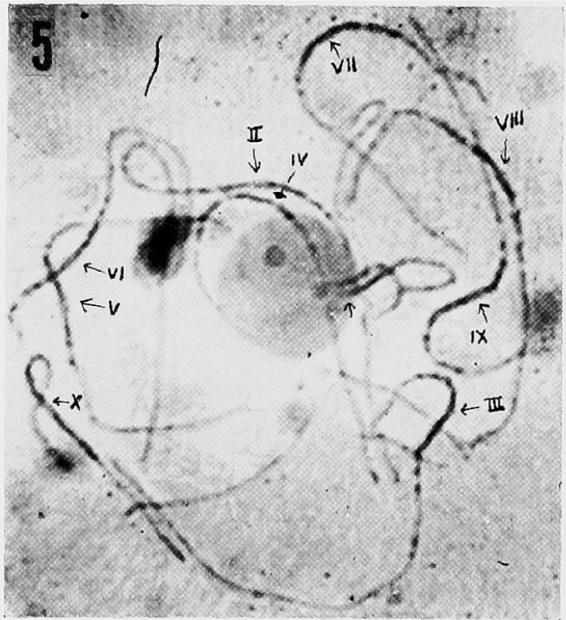

Fig. 5. Pachytene chromosome of S. dochna (ca. $\times 1000)$. ing the pycnotic region.

Chromosome III : Measures about $57.82 \mu$. Though this chromosome largely coincides with chromosome II in its total length, it can be separated from the later on the basis of arm ratio which is almost $1: 2$. The short arm has a very short darkly staining region which is followed by two chromomeres. On the long arm following the comparatively longer pycnotic region there are 2-3 chromomeres.

Chromosome IV: Measures about $52.88 \mu$ with an arm ratio of $1: 1.5$. The darkly staining region on the short arm ends with a chromomere close to it. But on the long arm following the proximal darkly staining region there is one more darkly staining region which is not comparable to a chromomere. However, this is shorter than the proximal pycnotic region.

Chromosome V: Unlike in some other species, this chromosome is more symmetrical in its form, having an arm ratio of $1: 1.1$ and measuring about $46.12 \mu$. The darkly staining regions on both the arms are almost equal but on one arm it ends abruptly and on the other it is followed by a chromomere. 
Chromosome VI: Measures about $36.72 \mu$. This is the most asymmetrical form of chromosome having an arm ratio of $1: 5$. The deeply staining region occupies about $3 / 4$ th of the short arm which is followed by 2-3 chromomeres. No chromomeres are noticed on the long arm following the pycnotic region.

Chromosome VII: Measures on an average $35.15 \mu$. Amongst the small chromosomes this can be easily picked out on the basis of an arm ratio which is $1: 1.7$ in addition to its characteristic feature with regard to pycnotic region. The short arm has a longer deeply staining region which ends abruptly whereas two chromomeres follow this region in the long arm.

Chromosome VIII : Measures about $32.81 \mu$ having an arm ratio of $1: 1.3$. This is very close to the chromosome IX in its total length but these two chromosomes can be differentiated on the basis of darkly staining region which are comparatively shorter on both arms in this chromosome than in chromosome IX. The deeply staining region on the short arm ends abruptly whereas on the long arm it is followed by a chromomere.

Chromosome IX: Measures about $31.25 \mu$ with an arm ratio of $1: 1.5$. The deeply staining region on the both arms is almost equal but longer than either in the 7 th or 8 th chromosome. On both the arms there are two chromomeres following the deeply staining region.

Chromosome $\mathrm{X}$ : This is the shortest chromosome of the complement measuring about $28.13 \mu$ and having an arm ratio of $1: 1.1$-a very symmetrical chromosome. It has a good amount of deeply staining region which on both the arms ends abruptly. The long arm ends with a terminal chromomere.

Based on the description of each chromosome given above (Table 1) the pachytene idiogram of all the ten pachytene bivalents in each these species has been constructed bringing out the diagnostic features of the individual chromosomes which proved particularly useful in their identification in the complement. Studies on the pairing properties of the differentially stained regions show that synapsis starts proximally to the centromere while separation of the split chromosomes is initiated from the distal and proceeds towards the centromere.

Diakinesis and metaphase I

The differentially stained regions were found to persist till late diakinesis in all these species. It is quite clear from the data presented in the Table 2 that there is no significant difference in chiasma frequency per cell between the species though there is decrease in the number of chiasmata per cell from early diakinesis to metaphase. The analysis made at early diakinesis in all these species showed that usually the longer chromosomes form a larger number of chiasmata (i.e. $3 \mathrm{xta} /$ bivalent) than the shorter ones (1 $\mathrm{xma}$ (bivalent). In a good number of cells, all the chromosomes appeared to have a minimum of 2 chiasmata. A critical analysis of the bivalents at dia- 
Table 2. Chiasma frequency in the five species at late diakinesis and metaphase I

\begin{tabular}{|c|c|c|c|c|c|c|}
\hline \multirow{2}{*}{ Species and stage } & \multirow{2}{*}{$\begin{array}{l}\text { Total no. } \\
\text { of cells }\end{array}$} & \multicolumn{3}{|c|}{ Bivalents with $2-0$ xta } & \multirow{2}{*}{$\begin{array}{c}\text { Total no. } \\
\text { of } x t a\end{array}$} & \multirow{2}{*}{$\begin{array}{c}\text { Average } \\
\text { no. of } \\
\text { xta/cell }\end{array}$} \\
\hline & & $2 x \operatorname{ta}$ & $1 \mathrm{xma}$ & 0xma & & \\
\hline $\begin{array}{l}\text { S. arundinaceum } \\
\text { late diakinesis } \\
\text { Metaphase I }\end{array}$ & $\begin{array}{l}25 \\
25\end{array}$ & $\begin{array}{l}219 \\
157\end{array}$ & $\begin{array}{l}31 \\
93\end{array}$ & $\begin{array}{l}0 \\
0\end{array}$ & $\begin{array}{l}469 \\
407\end{array}$ & $\begin{array}{l}18.76 \\
16.24\end{array}$ \\
\hline $\begin{array}{l}\text { S. verticilliflorum } \\
\text { late diakinesis } \\
\text { Metaphase I }\end{array}$ & $\begin{array}{l}25 \\
25\end{array}$ & $\begin{array}{l}237 \\
131\end{array}$ & $\begin{array}{r}13 \\
119\end{array}$ & $\begin{array}{l}0 \\
0\end{array}$ & $\begin{array}{l}487 \\
381\end{array}$ & $\begin{array}{l}19.48 \\
15.24\end{array}$ \\
\hline $\begin{array}{l}\text { S. caudatum } \\
\text { late diakinesis } \\
\text { Metaphase I }\end{array}$ & $\begin{array}{l}25 \\
25\end{array}$ & $\begin{array}{l}228 \\
129\end{array}$ & $\begin{array}{r}22 \\
121\end{array}$ & $\begin{array}{l}0 \\
0\end{array}$ & $\begin{array}{l}478 \\
379\end{array}$ & $\begin{array}{l}19.12 \\
15.16\end{array}$ \\
\hline $\begin{array}{l}\text { S. saccharatum } \\
\text { late diakinesis } \\
\text { Metaphase I }\end{array}$ & $\begin{array}{l}25 \\
25\end{array}$ & $\begin{array}{l}205 \\
155\end{array}$ & $\begin{array}{l}45 \\
95\end{array}$ & $\begin{array}{l}0 \\
0\end{array}$ & $\begin{array}{l}455 \\
405\end{array}$ & $\begin{array}{l}18.20 \\
16.20\end{array}$ \\
\hline $\begin{array}{l}\text { S. dochna } \\
\text { late diakinesis } \\
\text { Metaphase I }\end{array}$ & $\begin{array}{l}25 \\
25\end{array}$ & $\begin{array}{l}224 \\
131\end{array}$ & $\begin{array}{r}26 \\
119\end{array}$ & $\begin{array}{l}0 \\
0\end{array}$ & $\begin{array}{l}474 \\
381\end{array}$ & $\begin{array}{l}18.96 \\
15.20\end{array}$ \\
\hline
\end{tabular}

kinesis revealed that chiasmata are absent or rarely present in the deeply staining region. Other meiotic stages are quite normal. As expected pollen stainability was high in all these species.

\section{Discussion}

Snowden (1935) classified the Sorghum on the basis of morphology into two broad sections viz. Para-Sorghum and Eu-Sorghum. Garber (1950) subdivided the genus Sorghum into six sub-genera on the basis of morphological characters as also on certain cytological features of the meiotic chromosomes such as chromosome stainability at diakinesis, position and morphology of the nucleolus organizing region and number of nucleolar chromosomes at pachytene. The taxonomic aspect and some possible lines of inter-relationship between species were also indicated by him. However, the differentiation of species, belonging to the Sub-genus Eu-Sorghum has so far been mainly based on morphological characters. Celarier (1958) modified Garber's classification by treating Sorghastrum as a distinct genus. He has also listed the chromosome number for many of the species in the genus Sorghum.

All the species belonging to the subsection Arundinacea, to which the five species studied here belong, have chromosome number $n=10$. Hence it is difficult to identify the different species on the basis of chromosome number. It, therefore, becomes desirable to investigate in detail the karyomorphology of different species with a view to find out the features, if any, that they can be conveniently used in differentiating the species and can, perhaps, be correlated to certain morphological characters. However, these would depend on the precise identification of each of the ten chromosomes of the haploid complement followed preferably by the location of some marker 
genes on each of the chromosome, a type of study which has been worked out extensively in Maize and its relatives.

Sharma and Bhattacharjee (1957) have presented idiograms for 27 different species of Sorghum on the basis of mitotic chromosomes. The idiograms drawn by them show the existence of marked variations between the species. Such studies, however, do not usually give complete information about the morphology of the chromosomes because of extreme condensation of the chromosomes at this stage. Nevertheless, some preliminary idea regarding the chromosomal variations can be obtained provided suitable techniques are used.

A critical analysis of the pachytene stage of meiosis can, however, provide a better opportunity to study their morphology in greater detail than analysis of mitotic chromosomes. It is from this point of view that this study of the pachytene chromosomes was undertaken in five species of $\mathrm{Eu}$ -

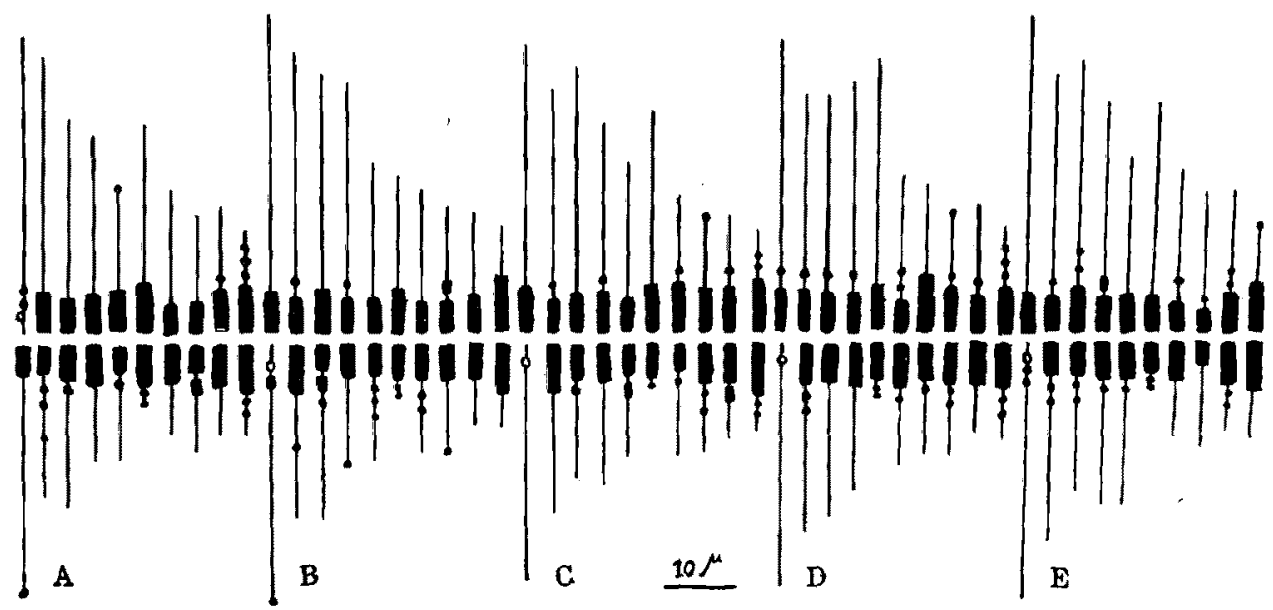

Fig. 6. The idiogram of the pachytene chromosomes of, A-S. arundinaceum; B-S. verticilliflorum; C-S. caudatum; D-S. saccharatum; and E-S. dochna. Chromosomes are arranged in the order of their length.

Sorghum since such information may be utilized in determining the relationship between these taxa. The difference between the pachytene idiograms of the species, if any, may give some information regarding the role of structural changes of chromosomes in the evolution of different species of Eu-Sorghum.

The study of pachytene chromosomes in these species and also in two species previously reported (see Magoon and Shambulingappa 1960) brings out great similarity in their morphology. However, some differences are also noticed between them. The nucleolar chromosomes in all these species has almost the same features. However, one marked difference that was observed with regard to the chromosome, in $S$. arundinaceum, is, that unlike in other species, here the nucleolar organizer is situated on the long arm. The fact 
that the morphology of the short arm in this species greatly resembles that of the long arm of the other species, suggests that the nucleolar organizer has not been transferred from the short arm but that this type of change may have been brought about by a pericentric inversion.

As regards chromosomes I, V, VI, VIII, IX and X, not much variation was observed except for the chromomeric pattern and slight variations in relative length. Chromosomes II, III, IV and VII show some variations in their arm ratio and the pattern of pycnotic regions as well as in relative length in the different species. Hence it appears that chromosomes I, V, VI, VIII, IX and $\mathrm{X}$ might not have undergone much structural alteration during the course of the differentiation of these closely related species.

Brown (1942) studied the meiosis in haploids of $S$. vulgare and reported the formation of 1-3 bivalents at MI. Later, Endrizzi and Morgan (1955) reported the presence of bivalents in a haploid plant obtained from a wide cross involving $S$. vulgare and $S$. arundinaceum. The later observations on derivatives of this haploid plant led the workers to postulate the existence of duplications of segments in the haploid complement of $S$. vulgare, an Eu-Sorghum. The segmental duplication in non-homologous chromosomes led them to suggest that the species concerned $S$. vulgare is an allo-and not an autopolyploid.

The karyotype of the species studied here, however, show almost similar features. However, the apparent absence of marked structural differences may be at least partly due to the inherent limitations of such studies.

If marked structural differences exist between different species it would be natural to expect some characteristic abnormalities in the meiosis in species hybrids. The preliminary observations made on some hybrids between the species concerned however, do not reveal any such abnormalities in meiosis (Magoon and Shambulingappa, unpublished data). However, detailed study of a large number of hybrids would be required before any definite conclusion can be arrived at. Though many other workers have also reported normal meiosis in interspecific crosses their observation were all from diakinesis and onwards (Endrizzi 1957, Krishnaswamy 1956). Such studies do not reveal the existence of small or "cryptic structural differences" between the species. Pachytene studies in hybrids between such species will definitely provide more reliable evidences on this point.

It has not been possible to get much information regarding the evolutionary process among the species studied here because of the great similarity of the karyotypes in the taxa. One interesting fact may, however, be noted. The wild species, $S$. arundinaceum and $S$, verticilliflorum are found to possess terminal chromomeres in the III and IV chromosomes while in the cultivated thus for studied, only one chromosome has this terminal chromomere. This agrees with the findings of Manglesdorf and Cameron (1942) who, in thẹir detailed study of the karyotype of races of maize and its rela- 
tives found that primitive types have more knobs.

Sharma and Bhattarcharjee (1957) have suggested on the basis of secondary association noted by them that the basic chromosome number in the genus is $n=4$. The exact significance of the phenomenon of secondary association has been controversial (see Magoon and Ramanujam 1960) and as such it may not be entirely sound to derive the basic number of a group of taxa on the basis of secondary association alone. Most of the other workers who have studied this genus feel that $n=5$ is the basic chromosome number in the genus. If this suggestion is accepted, then the whole haploid complement may be divided into two groups. Long (I-IV) ond short ( $\mathrm{V}$ to $\mathrm{X}$ ) in the present study. This suggests the possibility that these species have arisen as a result of hybridization between a 5-chromosomed form with long chromosomes and another 5 chromosomed form with short chromosomes followed by doubling of chromosome number in the hybrid, a situation reminiscent of the origin of the tetraploid cottons of the New World. Of course, only chromosome I-IV have been grouped as long chromosomes in this study but it may be pointed out that chromosome $\mathrm{V}$ is only relatively short as compared with these long chromosomes. Also, it may be that such short chromosomes may arise from a long chromosome due to chromosomal aberration. Through further evolutionary and selective mechanism the present type of karyotype may have been established. The later alterations in chromosomes which gave rise to different species and races may be mostly of the nature of "cryptic" structural changes.

Brown (1949) and Barton (1951) reported localization of chiasmata in tomato in the achromatic region. Venkateswaralu and Reddi (1956) have, on the basis of the assumption that chiasmata are randomly distributed over the entire chromosome complement, suggested that in the short arm of chromosome VI which is largely constituted of deeply staining regions, a great proportion of chiasmata should be occurring in this region. This is not supported by cytological observations, nor does it take into account the possibility of genetically determined localization of chiasmata to the ends of the chromosomes. In the present material, however, as in S. ankolib (Magoon and Shambulingappa 1960) microscopic observations during diakinesis have clearly shown that chiasmata are very seldom formed in the chromatic regions. If there is such a localization it would mean recombination of genes located on the heavily stained region would be highly restricted. The mode of pairing is another point that needs to be considered. Pairing may be initiated near the centromeric region or it may start at the distal end and proceed towards the centromere. Thus Brown (1949) in tomato found that pairing is initiated during zygotene or early pachytene stage in the distal achromatic zones followed later by pairing in the chromatic zones. Hyde (1953) in Plantago ovata found that pairing is initiated regularly in the middle segments while separation of the divided chromosomes appear first 
in the end segments during the early diplotene stage. Darlington (1933 and 1935) showed that in Fritillaria and Agapanthus initial pairing as well as diplotene separation takes place in the condensed proximal regions. In the present material as well as in Sorghum ankolib (Magoon and Shambulingappa 1960) the initiation of pairing is in line with that seen in Fritillaria and Agapanthus (see Darlington 1937) but the separation of the split chromosomes starts at the distal end as in Plantago ovata. Incidentally, the latter observation is in contrast to the findings of Venkateswaralu and Reddi (1956) in S. subglabrascens.

\section{Summary}

Detailed analysis of chromosomes at pachytene and later stages of meiosis were made in five species of Sorghum belonging to the Sub-genus $\mathbf{E u}$ Sorghum. Pachytene chromosomes are depicted on the basis of relative length, centromeric position, arm ratio and number of chromosomes which are adjoining the darkly staining region. The idiogram of all the five species which is represented diagramatically do not exhibit much variation. Pachytene chromosomes exhibit differentially staining regions which persists till late diakinesis. The nucleolus organizer in all the Eu-sorghums so far studied, is situated on the short arm of the first chromosome close to the centromere except for the species $S$. arundinaceum were it is located on the long arm. The possible cause for such difference is discussed.

Studies on the pairing properties of the differentially stained regions show that synapsis starts from proximal to the distal end and separation of split chromosomes starts from the distal and proceeds to the proximal. The distribution of chiasmate at different stages was studied. The average frequency of chiasmata per cell between different species show no significant difference. A critical analysis of the bivalents at diakinesis revealed that chiasmata are absent or rarely present in the deeply staining region. If such a localization prevails, it would mean, recombination of the genes located in the heavily stained regions would be highly restricted, to the non-staining regions. Based on the idiogram of the pachytene chromosomes the possible role of chromosomes in speciation and the relationship between species are discussed.

\section{Acknowledgement}

The authors wish to express their sincere gratitude to Dr. B. P. Pal, Director I. A. R. I. New Delhi and Dr. A. B. Joshi, Dean of the Postgraduate School, I. A. R. I., New Delhi, for their keen interest and constant encouragement. We are also grateful to Dr. M. S. Swaminathan and Shri S. Ramanujam for critically going through the maunscript. Co-operation from Dr. K. O. Rachie, Rockefeller Foundation, Botany Division, I. A. R. I., 
is also hereby acknowledged. One of us (K. G. S.) is also grateful to the University of Mysore for the award of a Scholarship during the course of this investigation.

\section{References}

Barton, D. W. 1950. Pachytene morphology of the tomato chromosome complement. Amer. J. Bot. 37: 639-43.

- 1951. Localized chiasmata in the differentiated chromosomes of the tomato. Genetics 36: $374-81$.

Brown, M. S. 1943. Haploid plants in Sorghum. J. Hered. 34: 163-166.

Brown, S. W. 1949. The structure and meiotic behaviour of the differentiated chromosomes of tomato. Genetics 34: 437-461.

Celarier, R. P. 1958. Cytotaxonomy of the Andropogoneae. III. Subtribe Sorgheae, Genus Sorghum. Cytologia 23: 395-418.

Darlington, C. D. 1933a. Meiosis in Agapantus and Kniphofia. Cytologia 4: 229-240.

- 1933b. The origin and behaviour of chiasmata. IX. Diploid and tetraploid Avena. Cytologia 5: 128-134.

- 1935. The internal mechanics of the chromosomes. III. Relational coiling and crossing over in Fritillaria. Proc. Roy. Soc. B, 118: 74-96.

- 1937. Recent advances in cytology. J and A. Churchill Ltd., London.

Endrizzi, J. E. 1957. Cytological studies of some species and hybrids in the Eu-sorghums. Bot. Gaz. 119: 1-10.

- and Morgan, D. T., Jr. 1955. Chromosomal interchanges and evidence for duplication, in haploid Sorghum vulgare. J. Hered. 46: 201-208.

Garber, E. D. 1948. Pachytene chromosomes of Sorghum intrans. J. Hered. 38: 251-252.

- 1950. Cytotaxonomic studies in the genus Sorghum. Univ. Cal. Publ. Bot. 23, No. 6: 283-362.

Gottschalk, W. 1954. Die Chromosomen Struktur der Solanaceen unter Berücksichtigungphylogenetischer Fragestellungen. Chromosoma 6: 539-626.

Huziwara, Y. 1956. Karyotype analysis in some genera of Compositeae. I. Karyotype of Japanese Eupatorium. Cytologia 21: 114-123.

Hyde, B. B. 1953 . Differentiated chromosomes in Plantago ovata. Amer. J. Bot. 44: 809-815.

Krishnaswamy, N., Raman, V. S. and Chandrashekharan, P. 1956. An interspecific hybrid of grain Sorghum and Johnson grass $S$. halepense $(2 \mathrm{n}=20) \times S$. roxburghii $(2 \mathrm{n}=20)$. Curr. Sci. 25: 195-197.

Magoon, M. L. and Shambhulingappa, K. G. 1960. Karyomorphological studies in Sorghum ankolib var. annalib red, A Eu-sorghum. Indian J. Genet. 20: 166-177.

-, Cooper, D. C. and Hougas, R. W. 1958. Cytogenetic studies of some diploid Solanums, section Tuberarium. Amer. J. Bot. 45: 207-221.

- and Ramanujam, S. 1960. Nature of chromosome pairing in the genus Solanum section Tuberarium. Indian J. Genet. 20: 22-41.

Mangelsdorf, P. C. and Cameron, J. W. 1942. Western Guatmale, a secondary centre of origin of cultivated maize varieties. Bot. Mus. Leal. Harv. 10: 217-256.

McClintock, B. 1929. Chromosome morphology in Zea mays. Science 69: 269.

Reddi, V. R. 1958. On the differentiation of A and B chromosomes of Sorghum purpureosericeum at Pachytene. J. Indian Bot. Soc. 37: 279-289.

Sharma, A. K. and (Miss) Dipti Bhattacharjee 1957. Chromosome studies in Sorghum. I. Cytologia 22: 287-311.

Snowden, J. D. 1935. The cultivated races of Sorghum. Adlard and Son., Ltd. 21 Hart Street, London, W.C.I. 
Swaminathan, M. S., Magoon, M. L. and Mehra, K. L. 1954. A simple propiono-carmine PMC. Smear method for plants with small chromosomes. Indian J. Genet. 14: 87-88.

Venkateswaralu, J. and Reddi, V. R. 1956. Morphology of the pachytene chromosomes and meiosis in Sorghum subglabrascens, a Eu-sorghum. J. Indian Bot. Soc. 35: 344-356. 